\title{
Toward Thing-to-Thing Optical Wireless Power Transfer: Metal Halide Perovskite Transceiver as an Enabler
}

\author{
Dinh Hoa Nguyen ${ }^{1,2 *}$, Toshinori Matsushima ${ }^{1,3}$, Chuanjiang Qin $^{3,4,5}$ and Chihaya Adachi ${ }^{1,3,5}$ \\ ${ }^{1}$ International Institute for Carbon-Neutral Energy Research (WPI-I2CNER), Kyushu University, Fukuoka, Japan, ${ }^{2}$ Institute of \\ Mathematics for Industry (IMI), Kyushu University, Fukuoka, Japan, ${ }^{3} J a p a n$ Science and Technology Agency (JST), ERATO, \\ Adachi Molecular Exciton Engineering Project, Fukuoka, Japan, ${ }^{4}$ State Key Laboratory of Polymer Physics and Chemistry, \\ Changchun Institute of Applied Chemistry (CIAC), Chinese Academy of Sciences, Changchun, China, ${ }^{5}$ Center for Organic \\ Photonics and Electronics Research (OPERA), Kyushu University, Fukuoka, Japan
}

OPEN ACCESS

Edited by:

Farhad Taghizadeh-Hesary,

Tokai University, Japan

Reviewed by: Sudhir Kumar,

ETH Zürich, Switzerland Chih-Jen Shih,

ETH Zürich, Switzerland

*Correspondence:

Dinh Hoa Nguyen

hoa.nd@i2cner.kyushu-u.ac.jp

Specialty section: This article was submitted to Sustainable Energy Systems and Policies,

a section of the journal Frontiers in Energy Research

Received: 11 March 2021 Accepted: 18 May 2021 Published: 17 June 2021

Citation:

Nguyen DH, Matsushima T, Qin C and Adachi C (2021) Toward Thing-toThing Optical Wireless Power Transfer: Metal Halide Perovskite Transceiver as an Enabler.

Front. Energy Res. 9:679125. doi: $10.3389 /$ fenrg.2021.679125
This paper proposes a novel conceptual system of optical wireless power transfer (OWPT) between objects, which is different from the existing OWPT systems such that a single device - an optical transceiver - is employed. This optical transceiver, which is capable of both absorbing and emitting light, is fabricated from a metal halide perovskite known for its superior features that can help significantly reduce the whole system size and cost. The proposed system contributes to realizing a thing-to-thing OWPT network, in which surfaces of objects/things are covered by perovskite transceivers (fully or partially), enabling them to wirelessly charge or discharge from the others.

Keywords: optical wireless power transfer, optical transceiver, metal halide perovskite, bidirectional wireless power transfer, wireless charging

\section{INTRODUCTION}

Recently, WPT has emerged as a promising and interesting approach for power supply without any cables or wires, due to many advantages it provides. First, WPT brings the convenience and comfort since we do not need to plug in wires/cables for charging our EVs or electronic devices, instead just park our EVs and put our devices near wireless charging equipment. Second, fire and explosion problems with wired charging can be avoided. Last but not least, WPT is a requisite for many scenarios where wired power transfer is expensive, difficult, dangerous, or impossible, e.g., in highvoltage power grids (Lu et al, 2018), space (NASA-USA, 2020a; JAXA-Japan, 2020), or deep water exploration missions. For example, very recently, a helicopter named Ingenuity, created by NASA-USA (2020b), was landed on Mars and will be tested to fly in Mars environment, which has a solar panel to get solar energy; hence, OWPT would be a promising solution to extend its exploration missions when solar energy is not directly available (see Figure $\mathbf{1}$ in Section $\mathbf{2}$ for an illustration).

In the literature, different WPT technologies have been investigated, for example, IWPT and resonant IWPT (Li and Mi, 2015; Hata et al., 2018; Patil et al., 2018; Machura and Li, 2019), CWPT (Mostafa et al., 2017), and OWPT (Fakidis et al., 2016; Yoshida et al., 2016; Jin et al., 2018; Katsuta and Miyamoto, 2018; Jin and Zhou, 2019), and have been employed in various realistic systems.

Abbreviations: CWPT, capacitive wireless power transfer; EL, electroluminescence; EQE, external quantum efficiency; EV, electric vehicle; IPCE, incident photon-to-electron conversion efficiency; IWPT, inductive wireless power transfer; LED, lightemitting diode; NIR, near-infrared; OWPT, optical wireless power transfer; PCE, power conversion efficiency; PL, photoluminescence; $\mathrm{QE}$, quantum efficiency; T2T, thing-to-thing; WPT, wireless power transfer. 


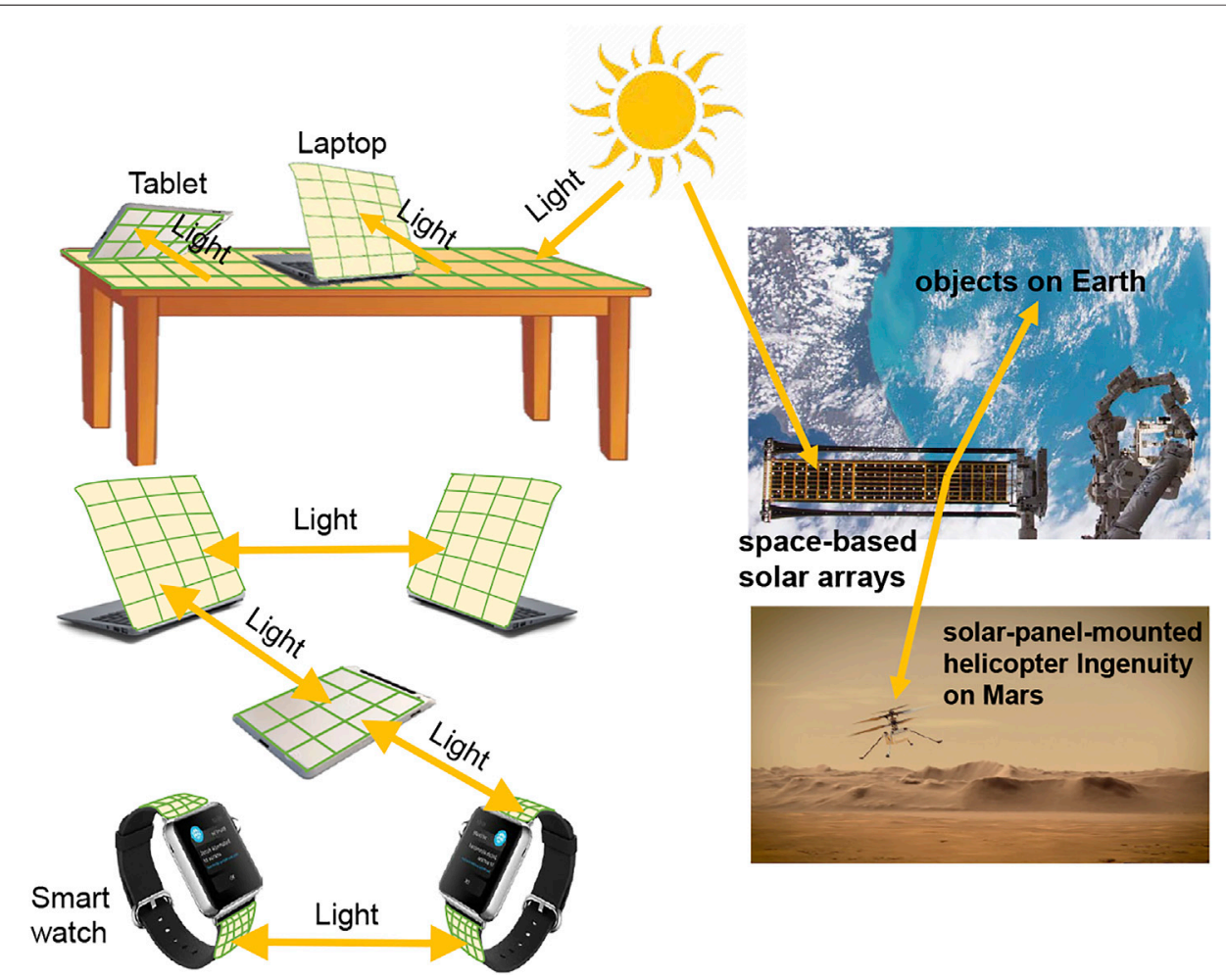

FIGURE 1 | Envisioned illustration of the proposed conceptual OWPT system toward realization of a T2T-OWPT network (e.g., table-laptop, table-tablet, laptop-laptop, tablet-watch, and watch-watch bidirectional OWPT).

Among those WPT technologies, resonant IWPT has the highest PCE, but its transfer distance is limited. The PCE of CWPT is quite high; however, it causes strong side effects to humans nearby. The transfer distance of CWPT is also short. For longer energy transfer distances, OWPT is one of the most suitable approaches; therefore, it has been studied extensively in both academia and industry, e.g., Caltech (2020); FISEGermany (2020); JAXA-Japan (2020); Wi-Charge (2020). In OWPT for long-distance applications (see, e.g., Fakidis et al., 2016; Katsuta and Miyamoto, 2018; Jin and Zhou, 2019), the laser beam is mostly used, but it is unsafe for humans, especially when exposed to eyes. On the contrary, LEDs are safer to be utilized in OWPT systems, but the generated light from them is more diverse than that from laser beams, leading to decreased efficiency and transfer distance. Hence, there is always a tradeoff between efficiency and safety in OWPT systems when using lasers and LEDs.

In most of the existing OWPT studies, both the wireless energy transmitter and receiver or one of them is stationary. Thus, the energy mobility and availability as well as the convenience and comfort of users have not been fully exploited. For instance, the proposed system in Wi-Charge (2020) can charge one or multiple devices as they are moving, but the transmitter is fixed on the ceiling; hence, those devices need to be kept around the transmitter. Our recent work (Nguyen, 2020) showcased a simple proof-of-concept for OWPT between objects that are all moving. Nevertheless, in all existing OWPT systems, whetherthe energy transfer is only unidirectional, or two separated devices (e.g., a solar panel and an LED in Nguyen (2020)) need to be utilized for each object for bidirectional energy transfer between them.

To overcome the above limitations of the existing OWPT studies, the current research proposes a novel, conceptual OWPT system, in which a metal halide perovskite transceiver is employed for both absorbing light from and emitting light to other OWPT systems equipped with similar perovskite transceivers. As a result, each OWPT system has a single optical transceiver for both transmitting and receiving energy, i.e., for performing bidirectional WPT. Furthermore, this concept allows the bidirectional OWPT to be conducted between different objects, whether they are stationary or in motion, because such system can be switched from the energy reception mode to the energy transmission mode anywhere, at any time. On the contrary, bidirectional WPT systems using other WPT technologies have recently been introduced, e.g., those using IWPT implemented in smartphones, but again, both objects need to be stationary. Note also that our proposed OWPT system is certainly able to harvest solar energy or energy from other lighting resources. Therefore, the issues of energy ultramobility and availability, user convenience, and user comfort can 
be addressed by our proposed perovskite-transceiver-based OWPT system. This system, if realized, will contribute a sustainable and green energy technology to the society.

It is worth noting that although our proposed OWPT system is conceptual, initial experimental results will be introduced to demonstrate such concept. To fully realize the proposed system, much more work is needed; however, it is promising since research progresses in perovskite solar cells and perovskite LEDs are very fast.

The rest of this paper is organized as follows. Section 2 introduces our proposed bidirectional OWPT using perovskite transceivers for realization of a T2T-OWPT network, remarkable properties of perovskite-based devices, and how they are used as optical transceivers in the proposed OWPT system, supported by experimental results and discussions on comparison with other WPT systems. Then, the overall system QE is characterized and its experimental results are presented in Section 3, together with its affecting factors. Lastly, Section 4 concludes our study and provides discussions on the usefulness of the proposed system, as well as directions for future research.

\section{PROPOSED OWPT SYSTEM AND RESULTS}

Figure 1 depicts the proposed OWPT system composed of basic elements such as devices whose surfaces are covered with optical transceivers and a transmission environment. The optical transceivers are made from a metal halide perovskite whose properties will be introduced in the next section. The transmission environment could be air, water, sea water, or outer space, depending on specific situations on which WPT is needed. Having the nice features of bidirectional transfer and ultra-mobility, the proposed OWPT system serves as an important step toward realization of the so-called T2T-OWPT network, in which energy can be ubiquitously exchanged anywhere and anytime between multiple objects/things equipped with perovskite transceivers, whether stationary or moving.

Note that the proposed conceptual OWPT system using a single optical transceiver for both emitting and absorbing light might be realized using other materials for the optical transceiver. For example, organic materials could be used, but their spectral overlap of light emission and absorption is small, i.e., the Stokes shift is large in organic materials. Meanwhile, it is currently difficult to find inorganic materials that can both emit and absorb light at high efficiency and low cost, like perovskites. Thus, a metal halide perovskite is proposed in the current research for fabricating optical transceivers.

On the contrary, it is certainly possible to create OWPT systems using separated devices for light emission and absorption (see, e.g., Hirukawa et al., 2019). However, in order to realize bidirectional OWPT between objects, both of those devices must be installed in every object, potentially leading to increased size, weight, and production cost and more complex electronic circuits. Additionally, those devices are not easy to be attached with curved surfaces. Hence, the current research proposes to use a single device-an optical transceiver made from a metal halide perovskite to overcome the above drawbacks. As a trade-off, the overall system efficiency of our proposed system is lower than those using separated devices for emitting and absorbing light, as will be discussed in more detail in Section 3.4.

To this end, it should be noted that the proposed OWPT system in this research is conceptual at this moment due to the limitation on the durability in the light emission mode of currently available perovskite solar cells, i.e., perovskite LEDs. However, the proposed system serves as the first step for a realistic realization of such OWPT systems in the future.

\subsection{Light Receiver Mode}

Metal halide perovskites have many advantages such as high carrier mobility, large carrier diffusion lengths, high absorption coefficients, bandgap tunability, thin and light weight, and compatibility with low-cost solution processing. These advantages make perovskites suitable for use in solar cells and can be flexibly attached on curved surfaces. Since the first discovery of perovskite solar cells in 2009 (Kojima et al., 2009), their performance has rapidly been increased. The highest certified PCE of perovskite solar cells has reached 25.5\% (National Renewable Energy Laboratory-USA, 2020) which is comparable to that of silicon solar cell technology. Our group in Kyushu University has investigated perovskite solar cells to improve their PCEs and operational durability (see, e.g., Qin et al., 2016; Qin et al., 2017; Qin et al., 2019; G. Tumen-Ulzii et al., 2020; Tumen-Ulzii et al., 2020). Our perovskite solar cells reported in Qin et al. (2019) had an architecture of glass substrate/indium tin oxide (ITO) electrode/poly(3,4-ethylenedioxythiophene):poly-

(styrenesulfonate) (PEDOT:PSS) hole transport layer/ $\mathrm{MA}_{0.6}$
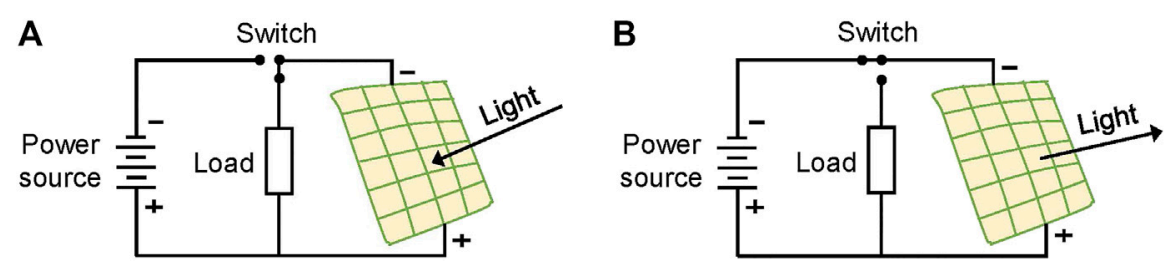

FIGURE 2 | Demonstrated circuit diagram of the proposed OWPT system with a perovskite transceiver: (A) receiver mode; (B) transmitter mode. 


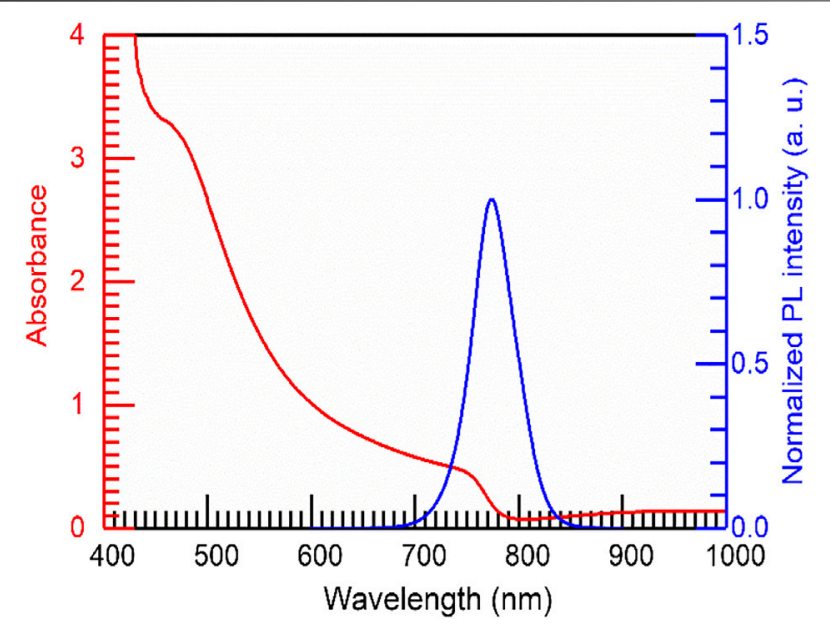

FIGURE 3 | Absorption and $\mathrm{PL}$ spectra of $\mathrm{MA}_{0.6} \mathrm{FA}_{0.4} \mathrm{Pbl}_{2.8} \mathrm{Br}_{0.2}$ perovskite films.

$\begin{array}{llll}\mathrm{FA}_{0.4} & \mathrm{PbI}_{2.8} & \mathrm{Br}_{0.2} & \text { perovskite light absorber }\end{array}$ methylammonium and $\mathrm{FA}$ is formamidinium) $/ \mathrm{C}_{60}$ electron transport layer/2,9-dimethyl-4,7-diphenyl-1,10-phenanthroline (BCP) electron transport layer/Au electrode.

In this light absorption working mode, the system operation is illustrated in Figure 2A. Note that what are shown in Figure 2 are just for illustration purposes, not exactly the same as in real systems. IPCEs of our perovskite solar cells are measured and will be discussed in the next section.

Detailed fabrication conditions of our $\mathrm{MA}_{0.6} \mathrm{FA}_{0.4} \mathrm{PbI}_{2.8} \mathrm{Br}_{0.2}$ perovskite films and solar cells are described in the Appendix. Although we already reported perovskite films and solar cells of the same composition in Qin et al. (2019), we briefly introduce their morphological, structural, and optical properties and solar cell performance as follows: $\mathrm{MA}_{0.6} \mathrm{FA}_{0.4} \mathrm{PbI}_{2.8} \mathrm{Br}_{0.2}$ perovskite films were polycrystalline, with grains of hundreds of nanometers in diameter, and had the pure tetragonal phase (Qin et al., 2019). The absorption onset wavelength is $\approx 780 \mathrm{~nm}$, and the photoluminescence (PL) peak wavelength is $\approx 775 \mathrm{~nm}$ (Figure 3). The PL quantum yield increases from $\approx 5$ to $\approx 22 \%$ as the excitation power is increased (Figure 4), probably because of the filling of defect states by photoexcited carriers or excited states in films. Time-resolved transient PL decay curves have a multiexponential decay behavior (Figure 5). The PL lifetime, at which $\mathrm{PL}$ intensity decreases to $1 / \mathrm{e}$ of the initial, is $\approx 35 \mathrm{~ns}$. $\mathrm{MA}_{0.6} \mathrm{FA}_{0.4}$

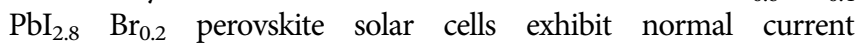
density-voltage curves under solar illumination (AM1.5G, $100 \mathrm{~mA} / \mathrm{cm}^{2}$ ), with a short-circuit current density of $20.14 \mathrm{~mA} / \mathrm{cm}^{2}$, an open-circuit voltage of $1.01 \mathrm{~V}$, a fill factor of 0.73 , and a PCE of $14.83 \%$, as reported in Qin et al. (2019).

\subsection{Light Transmitter Mode}

Perovskites are known to have the large spectral overlap between emission and absorption, as shown in Figure 3. When a high voltage is applied to a perovskite solar cell, electrons and holes were injected from electrodes and recombined in the perovskite

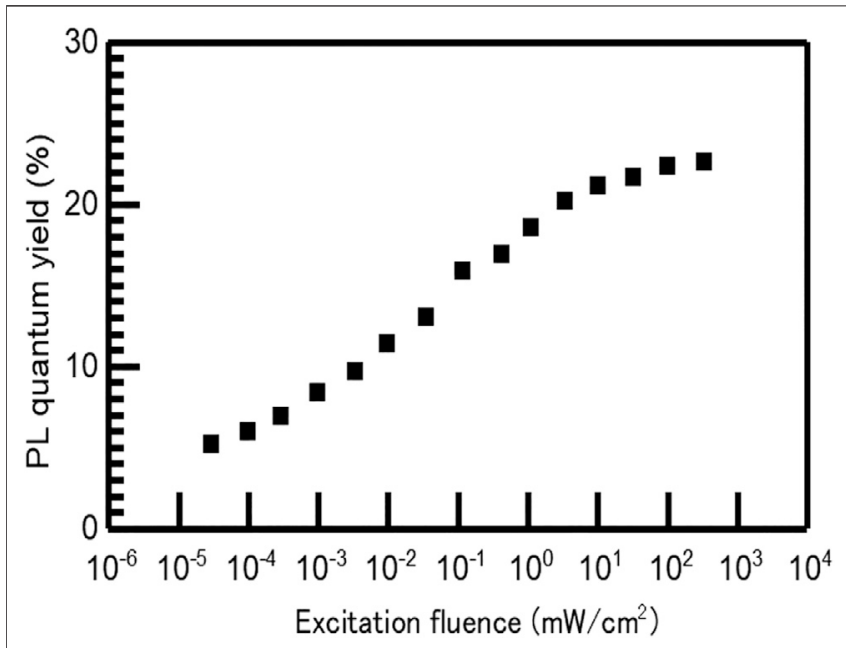

FIGURE 4 | Plot of PL quantum yields as a function of excitation fluence for $\mathrm{MA}_{0.6} \mathrm{FA}_{0.4} \mathrm{Pbl}_{2.8} \mathrm{Br}_{0.2}$ perovskite films.

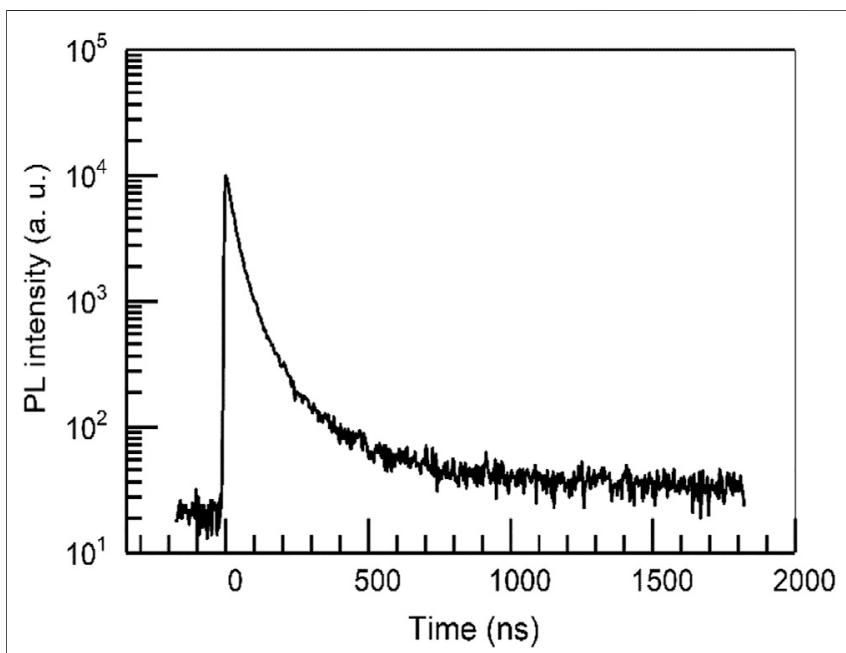

FIGURE 5 | Time-resolved transient PL curve of $\mathrm{MA}_{0.6} \mathrm{FA}_{0.4} \mathrm{Pbl}_{2.8} \mathrm{Br}_{0.2}$ perovskite films.

layer, which is a completely opposite process compared with that in solar cells. As a result, the perovskite solar cells began to emit light, i.e., they started working as light transmitters. Therefore, by taking advantage of efficient photoluminescence, perovskites can be employed as the emitter of efficient LEDs, i.e., a light transmitter in an OWPT system. Recently, very high EQEs of over $20 \%$ have been demonstrated from perovskite LEDs, see, e.g., Cao et al. (2018); Li et al. (2018); Xu et al. (2019). The operation principle of our proposed OWPT in the light-emitting mode is demonstrated in Figure 2B.

To make our perovskite solar cell emit light, a voltage is applied to it as mentioned above, which can be adjusted 


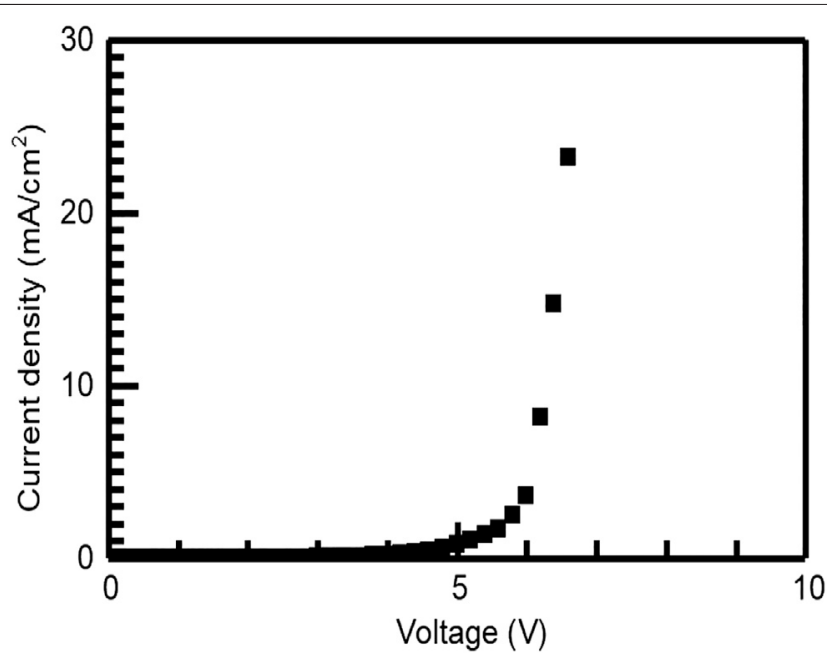

FIGURE 6 | Current density-voltage curve of $\mathrm{MA}_{0.6} \mathrm{FA}_{0.4} \mathrm{Pbl}_{2.8} \mathrm{Br}_{0.2}$ perovskite solar cells operating in the light-emitting mode.

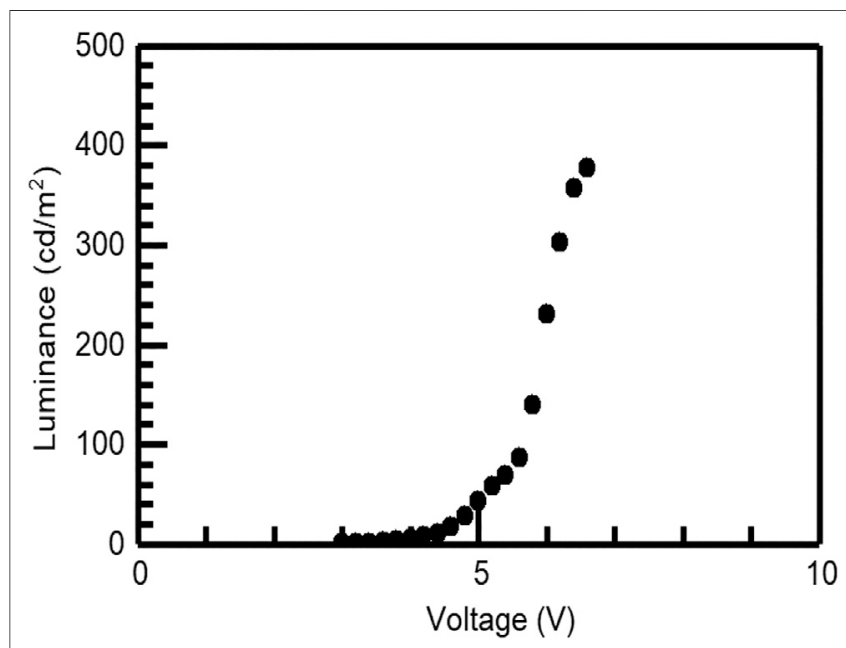

FIGURE 7 | Luminance-voltage curve of $\mathrm{MA}_{0.6} \mathrm{FA}_{0.4} \mathrm{Pbl}_{2.8} \mathrm{Br}_{0.2}$ perovskite solar cells operating in the light-emitting mode.

stepwise until obtaining the light emission (see Figure 6). The driving voltages to induce EL are below $10 \mathrm{~V}$, as depicted in Figures 6, 7. Then, Figure 8A displays a photograph of our $2 \mathrm{~mm}$ $\times 2 \mathrm{~mm}$ perovskite solar cell emitting NIR light under electrical excitation, which was used in our experiments. This emission of NIR light is useful for OWPT systems because NIR light is invisible and not so harmful to humans compared with other higher energy lights such as ultraviolet or visible lights.

As displayed in Figure 8B, our perovskite solar cell exhibits an EL peak at $790 \mathrm{~nm}$, which overlaps with the IPCE curve. Moreover, this perovskite cell shows light emission near the band edge. Emission at longer wavelengths is possible by utilizing smaller bandgap perovskites such as tin iodide-based perovskites (P. Šcajev et al., 2019). These features clearly show that perovskites can be utilized as both the light absorber of solar cells (optical receivers for OWPT systems) and the emitter of LEDs (optical transmitters for OWPT systems) simultaneously in a single-device architecture, i.e., an optical transceiver.

Note, however, that one limitation of all perovskite LEDs so far is their limited durability. This certainly affects the operation of perovskite-based optical transceivers, but we believe that the durability of perovskite LEDs will be significantly improved soon. In what follows, we demonstrate the optical wireless energy transmission between two identical perovskite transmitters. First, a perovskite transmitter is operated in the light-emitting mode, where pulsed voltages of $7 \mathrm{~V}$ with a width of $10 \mathrm{~ms}$ and a duty cycle of $50 \%$ are used to suppress the device degradation. Consequently, the emitted EL from that perovskite transmitter is detected by a silicon photodiode combined with an oscilloscope, which is exhibited in Figure 9. Second, another perovskite transmitter is placed in front of the first perovskite transmitter above instead of the silicon photodiode to detect EL. The distance between two perovskite transmitters is $\approx 1 \mathrm{~cm}$. A resistor with $50 \Omega$ is then placed in between the second perovskite transmitter and an oscilloscope. With this low resistance, the operation condition of the second perovskite transmitter nearly corresponds to the short-circuit condition. The short-circuit current coming from it is modulated in the manner similar to the EL response, as depicted in Figure 9. These results clearly indicate the possibility of performing the optical energy transmission (including optical communication) between two perovskite transmitters as we proposed.

\section{EFFICIENCY OF PROPOSED OWPT SYSTEM}

\subsection{Computation of System QE}

The OWPT system QE, denoted $\mathrm{EQE}_{\text {sys }}$, is derived by

$$
\mathrm{EQE}_{s y s}=\mathrm{EQE}_{L E D} \times \eta_{\text {env }} \times \mathrm{IPCE}_{P V},
$$

where $\mathrm{EQE}_{L E D}$ and $\mathrm{IPCE}_{P V}$ represent the QE of the optical transmitter and the optical receiver, respectively, while $\eta_{e n v}$ indicates the efficiency when light is transmitted through a specific environment.

In the current context of computing system $\mathrm{QE}, \eta_{\text {env }}$ is the ratio between the number of photons that reach the optical receiver and the number of photons emitted by the optical transmitter. If the wireless energy transmission distance is increased, the value of $\eta_{e n v}$ will be reduced, due to the spread of LED light beams, leading to that the less number of photons can reach the optical receiver. As a result, the overall system quantum efficiency is decreased at higher wireless energy transmission distances.

$\mathrm{EQE}_{L E D}$ and $\mathrm{IPCE}_{P V}$ are measured in our experiments and will be reported in the next section. Note that the overall system QE $\left(\mathrm{EQE}_{s y s}\right)$ in the current research is taken at a certain range of wavelengths, not over the whole solar light spectrum as for the system PCE. Hence, $\mathrm{EQE}_{s y s}$ is varied with different light wavelengths, due to the variation of the $\operatorname{IPCE}_{P V}$ of perovskite solar cells over the light spectrum. 

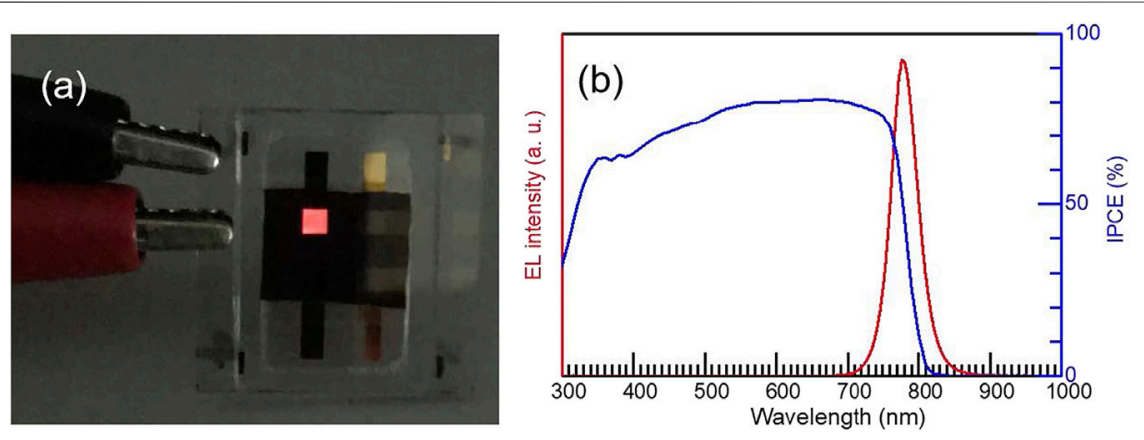

FIGURE 8 | (A) Photograph of an NIR light-emitting $\mathrm{MA}_{0.6} \mathrm{FA}_{0.4} \mathrm{Pbl}_{2.8} \mathrm{Br}_{0.2}$ perovskite solar cell. (B) EL spectrum and IPCE curve of the same device.

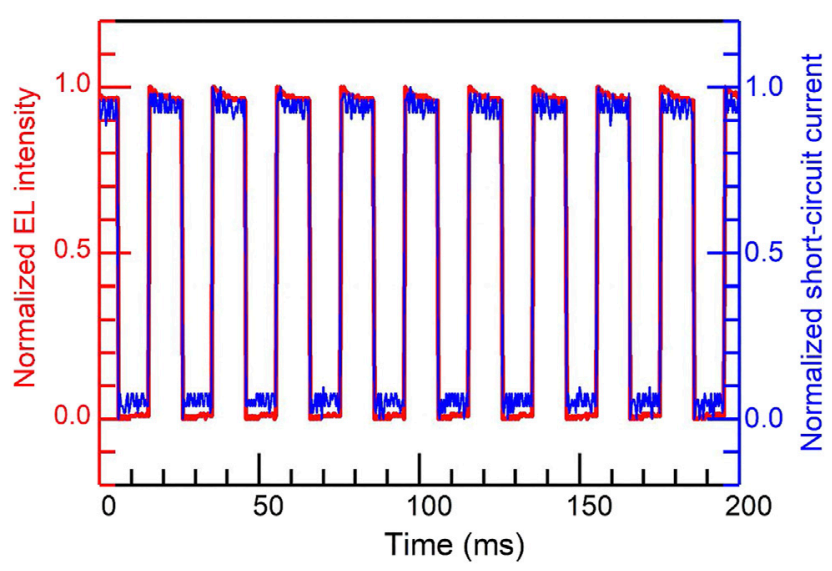

FIGURE 9 | Responses of normalized EL intensities and short-circuit current densities.

Remark 1: If PCE is considered for the proposed perovskitetransceiver-based OWPT system, then $\eta_{\text {env }}$ should be computed as the DC optical gain of LEDs (see Section 3.3 for more details). Moreover, the PCE of perovskite LEDs is not usually improved at the same time with the EQE of such devices due to their thickness (Miao et al., 2019). Therefore, one may have to balance between EQE and PCE for perovskite LEDs.

\subsection{Experimental QEs of Perovskite Transceiver}

The experimental $\mathrm{IPCE}_{P V}$ values of our perovskite solar cells are shown in Figure $8 B$, which is $46 \%$ at the EL peak wavelength and is highest of $\approx 80 \%$ at the wavelength of $740 \mathrm{~nm}$. The $\mathrm{EQE}_{L E D}$ value of the same solar cell working in the light-emitting mode is still low at $\approx 1 \%$ on average, as depicted in Figure 10. We need further study to increase its $\mathrm{EQE}_{L E D}$ in the future. It is interesting to note that the existing solar cells with high $\operatorname{IPCE}_{P V}$ values have low $\mathrm{EQE}_{L E D}$ values when they work as LEDs, and vice versa; those with high $\mathrm{EQE}_{L E D}$ values when operating as LEDs have low $\mathrm{IPCE}_{P V}$ values in the solar cell operation mode (Rau, 2007).

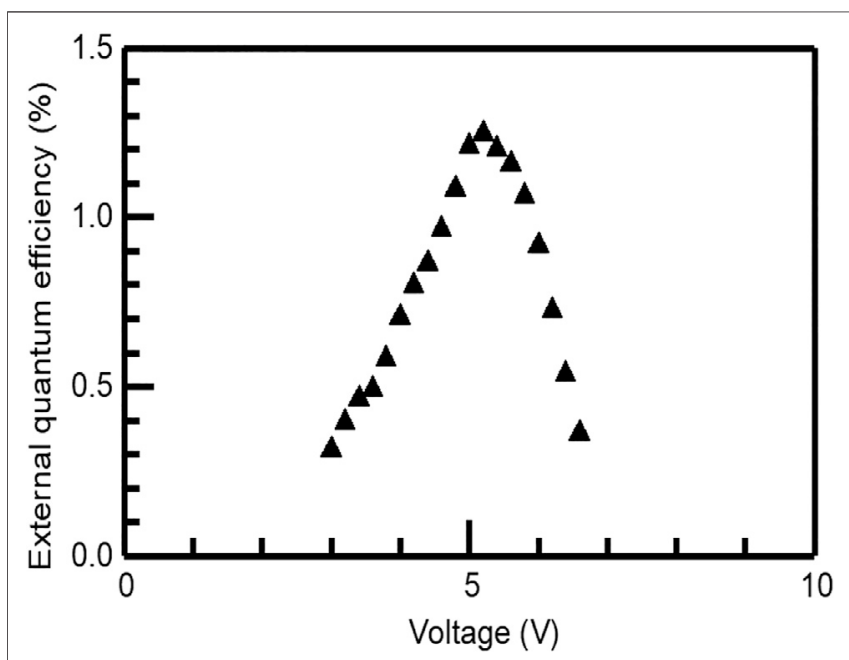

FIGURE 10 | EQE-voltage curve of $\mathrm{MA}_{0.6} \mathrm{FA}_{0.4} \mathrm{Pbl}_{2.8} \mathrm{Br}_{0.2}$ perovskite solar cells.

Consequently, $\mathrm{EQE}_{s y s}$ of the whole bidirectional OWPT system based on our metal halide perovskite transceiver, at the $790 \mathrm{~nm}$ wavelength at which the EL intensity is highest and at zero transmission distance, computed according to Eq. (1), is $0.46 \%$ at present. On the contrary, the highest $\mathrm{EQE}_{L E D}$ reported for NIR perovskite LEDs to date is $21.6 \%$ (Xu et al., 2019). If a similar $\mathrm{EQE}_{L E D}$ value can be obtained together with our reported IPCE $_{P V}$ value, then the overall perovskite-transceiver-based OWPT system quantum efficiency $\mathrm{EQE}_{s y s}$ is increased to $9.94 \%$ at the $790 \mathrm{~nm}$ light wavelength and at zero transmission distance. Nevertheless, as noted above, achieving a solar cell with high values of both $\mathrm{EQE}_{L E D}$ and $\mathrm{IPCE}_{P V}$ is not available at the moment but would be in the future. It should also be noted that some special methods in the literature, for example, those presented in Gets et al. (2019); Gets et al. (2021), could be employed to enhance the operation of perovskite transceivers.

Detailed reciprocal relations between EQE and EL emission were shown in Rau (2007) with rigorous mathematical derivations, which are useful for further studying and 
improving the optical transceivers in OWPT systems. In particular, the direct relation between $\mathrm{IPCE}_{P V}$ and EL emission was revealed in Rau (2007, Eq. 29) and Rau (2007, Eq. 6, 7).

At higher wireless energy transmission distances, the value of $\eta_{\text {env }}$ will be reduced, as mentioned above. Unfortunately, the state-of-the-art perovskite LEDs have not been durable enough for a long time for experimentally verifying such efficiency calculation, as mentioned above in Section 2.2. Thus, we have not obtained experimental results for higher energy transmission distances to show here and have left them for future reports.

\subsection{Calculation of System PCE}

When perovskite LEDs are stable enough (in the future), similar experiments like those reported in Nguyen (2020) using usual LEDs can be conducted to verify how the overall perovskitetransceiver-based OWPT system PCE depends on the transmitting distance. In what follows, we briefly revisit the theoretical and practical ways to derive the PCE of OWPT.

Theoretically, the PCE of an OWPT system, denoted $\mathrm{PCE}_{s y s}$, is calculated by

$$
\mathrm{PCE}_{s y s}=\mathrm{PCE}_{t r} \times \zeta_{e n v} \times \mathrm{PCE}_{r c},
$$

where $\mathrm{PCE}_{t r}$ and $\mathrm{PCE}_{r c}$ represent the PCE of the optical transmitter and receiver, respectively, and $\zeta_{\text {env }}$ indicates the optical power transmission efficiency through an environment (e.g., air, pure water, and sea water). For simplicity, let us consider the optical transmission through the air, then $\zeta_{\text {env }}$ is equal to the DC gain of the optical transmissions link that can be mathematically characterized as follows (Komine and Nakagawa, 2004; Nguyen, 2020):

$$
\zeta_{\text {env }}= \begin{cases}\frac{\left(m_{l}+1\right) A}{2 \pi d^{2}} \cos ^{m_{l}} \phi g_{f}(\varphi) g_{c}(\varphi) \cos \varphi, & 0 \leq \varphi \leq \varphi_{w} \\ & 0, \varphi>\varphi_{w}\end{cases}
$$

in which $A$ is the physical area of the optical receiver, $d$ is the optical transmission distance, $\varphi$ is the angle of incidence (i.e., the angle at which the receiver sees the transmitter), $\phi$ is the angle of irradiance (i.e., the angle at which the transmitter sees the receiver), $g_{f}(\varphi)$ is the gain of an optical filter if exists, $g_{c}(\varphi)$ is the gain of an optical concentrator if exists, and $\varphi_{w}$ is the width of the field of view (FOV) at the receiver. Moreover, $m_{l}$ denotes the order of Lambertian emission that is given by the semi-angle at half illuminance $\Phi_{1 / 2}$ of an LED as follows:

$$
m_{l}=-\frac{\ln 2}{\ln \left(\cos \Phi_{1 / 2}\right)} \text {. }
$$

We can clearly see from Eq. 3 that once OWPT happens, $\zeta_{e n v}$, and hence $\mathrm{PCE}_{s y s}$, is inversely quadratically decreased as the transmission distance is increased.

In practice, $\mathrm{PCE}_{s y s}$ can be obtained by taking the ratio of measured input power at the transmitter and measured output power at the receiver, similarly to what was reported in Nguyen (2020). It is unfortunate, as mentioned in Section 3.2, that perovskite solar cells are currently not durable enough in the LED mode to do many experiments; therefore, we could not experimentally verify the $\mathrm{PCE}_{S Y S}$ of the proposed OWPT system concept as the transmitting distance is changed. We are currently working to improve the durability of perovskite LEDs and hope to present such results in a near future report.

\subsection{Affecting Factors to System Efficiency}

First, system efficiency (whether QE or PCE) is affected by the alignment between the light transmitter and the light receiver, where any misalignment can make system efficiency decrease. Note that this happens to not only OWPT systems but also other WPT systems such as IWPT or CWPT. However, in such scenarios for OWPT systems, wireless energy transmitters based on LEDs are better than those based on lasers because OWPT might still occur using LEDs, thanks to their spread light beams, while it does not happen with lasers having focused and narrow light beams. To overcome the drawback of misalignment in WPT systems, additional devices are needed to help the energy transmitter and receiver track and align with each other. Nonetheless, this will certainly increase the system complexity, size, weight, and cost.

To quantitatively verify how the alignment between perovskite solar cells affects the overall system efficiency, additional experiments need to be conducted. Again, as discussed before, currently available perovskite LEDs are not durable enough for doing many experiments; hence, we hope to be able to report such results in the near future.

Next, it is well known that the light beams of LEDs are spread, unlike those of lasers, which reduces the overall system efficiency since less energy can reach the light receiver when traveling through the transmission range. Recent progresses in perovskite laser research [see, e.g., reviews in Quan et al. (2019); Dong et al. (2020); Gunnarsson and Rand (2020), and our recent work by Qin et al. (2020)] suggest that electrically driven lasers based on a metal halide perovskite emitter are promising and hence can be employed for our proposed perovskite-transceiver-based bidirectional OWPT system to increase the overall system efficiency. Nevertheless, there are also disadvantages of lasers compared to LEDs; one is the unsuccessful WPT if misalignment between the optical transmitter and receiver exists, as noted above, and the other is the safety concern of lasers. Another solution to increase the system efficiency without using lasers is to utilize supplemented devices to spread the LED light beams less (see, e.g., Song et al., 2018), but such devices will certainly make the OWPT system more complicated and costly.

Lastly, energy losses, caused by load resistance in the wireless energy-receiving devices, can reduce system efficiency if the load resistance is considerably high. However, this not only happens to our proposed OWPT systems but also occurs in other WPT systems. Details on this issue should be investigated in a separate study; hence, we leave it as future work.

\section{CONCLUSION AND DISCUSSION}

\subsection{Summary}

This article has proposed a novel conceptual OWPT system between objects equipped with optical transceivers made from metal halide perovskite devices. Each of such objects can work as 
both a light absorber and a light emitter, leading to bidirectional OWPT between those objects, whether they are stationary or moving. Theoretical characterization of the overall system energy conversion efficiency has been provided, and experimental results on the light absorption and emission modes of perovskite devices have been reported.

Based on the experimental results and those reported in the literature, the QE of the proposed perovskite-transceiver-based OWPT system is now maximally $9.94 \%$ at the $790 \mathrm{~nm}$ light wavelength (peak EL intensity). The PCE of the proposed system is anticipated to be much lower than that of the resonant IWPT system. However, the payoffs of rather simple electronic requirement, small size and weight, potentially low cost, usability for curved surfaces, printability, and full support for moving objects render the proposed OWPT system appropriate for ubiquitous applications, especially in the current Internet-of-things (IoT) era. Those applications include not only the wireless energy transfer between devices equipped with perovskite transceivers but also the harvest of solar energy and energy from other lighting resources. Hence, this perovskite-based OWPT system provides a sustainable and green energy technology approach to the society.

In addition, the proposed conceptual system contributes the first step toward realization of a T2T-OWPT network, in which OWPT can be made anywhere and anytime between diverse entities (things), e.g., consumer electronic devices, implant medical devices, robots, and IoT devices. Such T2T-OWPT network will bring not only the ultra-mobility of energy but also the convenience and comfort for humans.

\subsection{Further Discussions}

The durability of perovskite LEDs is an important issue needed to be improved in the near future in order to make them available for commercialization as well as for reliable serving in the proposed OWPT system. Furthermore, additional devices such as the optical reflectors and concentrators can be employed to increase the overall system energy conversion efficiency.

In the area of vehicle electrification, our proposed OWPT system could impinge upon novel wireless charging and discharging methods for electrified vehicles covered with perovskite solar cells. It is worth mentioning that solar-cell-covered vehicles have recently been initiated by several automobile companies, e.g., Hyundai (2020); NEDO-Sharp-Toyota (2020); Sion (2020).

It is also worth noting that the laser can be used in the proposed perovskite-transceiver-based bidirectional OWPT system, in which the laser beam is generated in the light-emitting mode instead of LED light. Due to the coherence of laser beams, higher overall system energy conversion efficiency and longer WPT range could be achieved. Although the laser beam is harmful to humans if the

\section{REFERENCES}

Caltech, U. (2020). Space Solar Power Project. [retrieved 15 October 2020], Available at: https://www.spacesolar.caltech.edu.

Cao, Y., Wang, N., Tian, H., Guo, J., Wei, Y., Chen, H., et al. (2018). Perovskite Light-Emitting Diodes Based on Spontaneously Formed Submicrometre-Scale Structures. Nature 562, 249-253. doi:10.1038/s41586-018-0576-2 energy level is high, as discussed before, this kind of system could be useful in specific applications where humans and other species are not present and energy grids do not exist or are not easily implemented, e.g., in space exploration missions (for instance, the Moon or Mars exploration) or in maritime applications. In such situations, other WPT technologies such as IWPT or CWPT are hard to be deployed, while with the proposed bidirectional perovskite-transceiver-based OWPT systems, solar energy can be harvested by spacecraft, satellites, landers, rovers, maritime vehicles and equipment, etc., and then is wirelessly transmitted to the others when they lack energy but cannot receive it from solar. As a result, the mission period can be extended smoothly, which is very important for such applications. Therefore, the proposed OWPT systems provide a viable solution despite some limitations (e.g., low efficiency, direct line-of-sight) as commonly found in other applications using solar cells.

Finally, light exposure safety would be of concern under high light intensity (e.g., when using the laser beam), or under high energy levels. For extensive information related to such issues, existing standards and guidelines are useful, see, e.g., ICNIRP (2020); Renesas (2020).

\section{DATA AVAILABILITY STATEMENT}

The original contributions presented in the study are included in the article/Supplementary Material, and further inquiries can be directed to the corresponding author.

\section{AUTHOR CONTRIBUTIONS}

DN conceptualized the idea, performed the methodology, and wrote the original draft. CQ and TM performed the experiments and reviewed and edited the paper. CA supervised the work and also took part in reviewing the paper.

\section{FUNDING}

The first author's research was partially funded by JSPS Kakenhi Grant Number JP19K15013. Additionally, this research was supported by the Japan Science and Technology Agency (JST), ERATO, Adachi Molecular Exciton Engineering Project (JST ERATO Grant Number JPMJER1305); JSPS KAKENHI (grant numbers JP16H04192 and JP20H02817); The Canon Foundation; and The Samco Foundation. 
FISE-Germany (2020). Science blog [retrieved 15 October 2020], Available at: https://blog.innovation4e.de/en/2016/09/12/power-by-light.

Gets, D., Alahbakhshi, M., Mishra, A., Haroldson, R., Papadimitratos, A., Ishteev, A., et al. (2021). Reconfigurable Perovskite Lec: Effects of Ionic Additives and Dual Function Devices. Adv. Opt. Mater. 9, 2001715. doi:10.1002/adom.202001715

Gets, D., Saranin, D., Ishteev, A., Haroldson, R., Danilovskiy, E., Makarov, S., et al. (2019). Light-emitting Perovskite Solar Cell with Segregation Enhanced Self Doping. Appl. Surf. Sci. 476, 486-492. doi:10.1016/j.apsusc.2019.01.031

Gunnarsson, W. B., and Rand, B. P. (2020). Electrically Driven Lasing in Metal Halide Perovskites: Challenges and Outlook. APL Mater. 8, 030902. doi:10.1063/1.5143265

Hata, K., Hanajiri, K., Imura, T., Fujimoto, H., Hori, Y., Sato, M., et al. (2018). Driving Test Evaluation of Sensorless Vehicle Detection Method for In-Motion Wireless Power Transfer, In The 2018 International Power Electronics Conference, 1-4.

Hirukawa, H., Yamaguchi, T., Ushida, Y., Onuma, T., and Honda, T. (2019). Prototype Optical Wireless Power Transmission System Using Blue Ld as Light Source and Led as Photovoltaic Receiver, In The 1st Optical Wireless and Fiber Power Transmission Conference. Yokohama, Japan: OWPT.

Hyundai (2020). [retrieved 15 October 2020], Available at: https://www.hyundai. news/eu/brand/hyundai-launches-first-car-with-solar-roof-charging-system.

ICNIRP (2020). Icnirp Guidelines on Limits of Exposure to Incoherent Visible and Infrared Radiation. [retrieved 15 October 2020], Available at: https://www. icnirp.org/cms/upload/publications/ICNIRPVisible_Infrared2013.pdf.

JAXA-Japan (2020). Research on Laser Wireless Power Transmission Technology. [retrieved 15 October 2020], Available at: http://www.kenkai.jaxa.jp/eng/ research/ssps/ssps-lssps.html.

Jin, K., and Zhou, W. (2019). Wireless Laser Power Transmission: A Review of Recent Progress. IEEE Trans. Power Elect. 34 (4), 3482-3499. doi:10.1109/tpel.2018.2853156

Jin, M. H. C., Pierce, J. M., Lambiotte, J. C., Fite, J. D., Marshall, J. S., and Huntley, M. A. (2018). Underwater Free-Space Optical Power Transfer: An Enabling Technology for Remote Underwater Intervention. Offshore Tech. Conf. 1-9. doi:10.4043/28892-MS

Katsuta, Y., and Miyamoto, T. (2018). Design and Experimental Characterization of Optical Wireless Power Transmission Using GaAs Solar Cell and SeriesConnected High-Power Vertical Cavity Surface Emitting Laser Array. Jpn. J. Appl. Phys. 57 (08PD01). doi:10.7567/jjap.57.08pd01

Kojima, A., Teshima, K., Shirai, Y., and Miyasaka, T. (2009). Organometal Halide Perovskites as Visible-Light Sensitizers for Photovoltaic Cells. J. Am. Chem. Soc. 131 (17), 6050-6051. doi:10.1021/ja809598r

Komine, T., and Nakagawa, M. (2004). Fundamental Analysis for Visible-Light Communication System Using LED Lights. IEEE Trans. Consumer Electron. 50 (1), 100-107. doi:10.1109/tce.2004.1277847

Li, S., and Mi, C. C. (2015). Wireless Power Transfer for Electric Vehicle Applications. IEEE J. Emerging Selected Topic Power Elect. 3 (1), 4-17.

Lin, K., Xing, J., Quan, L. N., de Arquer, F. P. G., Gong, X., Lu, J., et al. (2018). Perovskite Light-Emitting Diodes with External Quantum Efficiency Exceeding 20 Per Cent. Nature 562, 245-248. doi:10.1038/s41586-018-0575-3

Lu, M., Bagheri, M., James, A. P., and Phung, T. (2018). Wireless Charging Techniques for Uavs: A Review, Reconceptualization, and Extension. IEEE Access 6, 29865-29884. doi:10.1109/access.2018.2841376

Machura, P., and Li, Q. (2019). A Critical Review on Wireless Charging for Electric Vehicles. Renew. Sust. Energ. Rev. 104, 209-234. doi:10.1016/j.rser.2019.01.027

Miao, Y., Ke, Y., Wang, N., Zou, W., Xu, M., Cao, Y., et al. (2019). Stable and Bright Formamidinium-Based Perovskite Light-Emitting Diodes with High Energy Conversion Efficiency. Nat. Commun. 10, 3624. doi:10.1038/s41467-019-11567-1

Mostafa, T. M., Muharam, A., and Hattori, R. (2017). Wireless Battery Charging System for Drones via Capacitive Power Transfer, In 2017 IEEE PELS Workshop on Emerging Technologies: Wireless Power Transfer, 1-6.

NASA-USA (2020a). 2020 nasa technology taxonomy [retrieved 15 October 2020], Available at: https://www.nasa.gov/sites/default/files/atoms/files/2020_nasa_ technology_taxonomy_lowres.pdf.

NASA-USA (2020b). 6 Things to Know about Nasa's Ingenuity mars Helicopter. [retrieved 6 February 2021], Available at: https://www.nasa.gov/feature/jpl/6things-to-know-about-nasas-ingenuity-mars-helicopter.

National Renewable Energy Laboratory-USA (2020). Best Research-Cell Efficiencies. [retrieved 15 October 2020], Available at: https://www.nrel.gov/ pv/assets/pdfs/best-research-cell-efficiencies.20200925.pdf.
NEDO-Sharp-Toyota (2020). [retrieved 15 October 2020], Available at: https:// global.toyota/en/newsroom/corporate/28787347.html.

Nguyen, D. H. (2020). Optical Wireless Power Transfer for Moving Objects as a Life-Support Technology, In The 2020 IEEE 2nd Global Conference on Life Sciences and Technologies. Kyoto, Japan LifeTech, 405-408.

Patil, D., Mcdonough, M. K., Miller, J. M., Fahimi, B., and Balsara, P. T. (2018). Wireless Power Transfer for Vehicular Applications: Overview and Challenges. IEEE Trans. Transp. Electrific. 4 (1), 3-37. doi:10.1109/tte.2017.2780627

Qin, C., Matsushima, T., Fujihara, T., and Adachi, C. (2017). Multifunctional Benzoquinone Additive for Efficient and Stable Planar Perovskite Solar Cells. Adv. Mater. 29 (4), 1603808. doi:10.1002/adma.201603808

Qin, C., Matsushima, T., Fujihara, T. W. J. P., Jr., Potscavage, W. J., and Adachi, C. (2016). Degradation Mechanisms of Solution-Processed Planar Perovskite Solar Cells: Thermally Stimulated Current Measurement for Analysis of Carrier Traps. Adv. Mater. 28 (3), 466-471. doi:10.1002/adma.201502610

Qin, C., Matsushima, T., Klotz, D., Fujihara, T., and Adachi, C. (2019). Device Stability: The Relation of Phase-Transition Effects and Thermal Stability of Planar Perovskite Solar Cells (Adv. Sci. 1/2019). Adv. Sci. 6 (1), 1970004. doi:10. 1002/advs.201970004

Qin, C., Sandanayaka, A. S. D., Zhao, C., Matsushima, T., Zhang, D., Fujihara, T., et al. (2020). Stable Room-Temperature Continuous-Wave Lasing in Quasi-2d Perovskite Films. Nature 585, 53-57. doi:10.1038/s41586-020-2621-1

Quan, L. N., Rand, B. P., Friend, R. H., Mhaisalkar, S. G., Lee, T.-W., and Sargent, E. H. (2019). Perovskites for Next-Generation Optical Sources. Chem. Rev. 119 (12), 7444-7477. doi:10.1021/acs.chemrev.9b00107

Rau, U. (2007). Reciprocity Relation between Photovoltaic Quantum Efficiency and Electroluminescent Emission of Solar Cells. Phys. Rev. B. 76, 085303. doi:10.1103/physrevb.76.085303

Renesas (2020). Eye Safety for Proximity Sensing Using Infrared Light-Emitting Diodes. (version 3.00, apr. 28, 2016) [retrieved 15 October 2020]. https://www. renesas.com/jp/ja/doc/application-note/an1737.pdf.

Šcajev, P., Aleksiejūnas, R., Baronas, P., Litvinas, D., Kolenda, M., Qin, C., et al. (2019). Carrier Recombination and Diffusion in Wet-Cast Tin Iodide Perovskite Layers under High Intensity Photoexcitation. The J. Phys. Chem. C 123 (32), 19275-19281.

Sion, S. (2020). [retrieved 15 October 2020], Available at: https://sonomotors.com/ en/sion. doi:10.1145/3372297.3416242

Song, J., Kim, K. H., Kim, E., Moon, C. K., Kim, Y. H., Kim, J. J., et al. (2018). Lensfree Oleds with over 50\% External Quantum Efficiency via External Scattering and Horizontally Oriented Emitters. Nat. Commun. 9, 3207. doi:10.1038/s41467-018-05671-x

Tumen-Ulzii, G., Matsushima, T., Klotz, D., Leyden, M. R., Wang, P., Qin, C., et al. (2020). Hysteresis-less and Stable Perovskite Solar Cells with a Self-Assembled Monolayer. Commun. Mater. 1 (31). doi:10.1038/s43246-020-0028-Z

Tumen-Ulzii, G., Qin, C., and Adachi, C. (2020). Detrimental Effect of Unreacted Pbi2 on the Long-Term Stability of Perovskite Solar Cells. Adv. Mater. 32 (16), 1905035.

Xu, W., Hu, Q., Bai, S., Bao, C., Miao, Y., Yuan, Z., et al. (2019). Rational Molecular Passivation for High-Performance Perovskite Light-Emitting Diodes. Nat. Photon. 13, 418-424. doi:10.1038/s41566-019-0390-x

Yoshida, S., Tanomura, M., Hama, Y., Hirose, T., Suzuki, A., Matsui, Y., et al. (2016). Underwater Wireless Power Transfer for Non-fixed Unmanned Underwater Vehicle in the Ocean. In 2016 IEEE/OES Autonomous Underwater Vehicles (AUV). 177-180.

Conflict of Interest: The authors declare that the research was conducted in the absence of any commercial or financial relationships that could be construed as a potential conflict of interest.

Copyright (๑) 2021 Nguyen, Matsushima, Qin and Adachi. This is an open-access article distributed under the terms of the Creative Commons Attribution License (CC $B Y)$. The use, distribution or reproduction in other forums is permitted, provided the original author(s) and the copyright owner(s) are credited and that the original publication in this journal is cited, in accordance with accepted academic practice. No use, distribution or reproduction is permitted which does not comply with these terms. 


\section{APPENDIX}

\section{Detailed Fabrication Conditions of $\mathrm{MA}_{0.6}$ $\mathrm{FA}_{0.4} \mathrm{PBI}_{2.8} \mathrm{BR}_{0.2}$ Perovskite Solar Cells and Films}

Glass substrates coated with a prepatterned ITO layer with a thickness of $\approx 150 \mathrm{~nm}$ (ATSUGI MICRO) or fused silica substrates were cleaned by ultrasonication in detergent, pure water, acetone, and isopropanol and then subjected to UV-ozone treatment.

For the perovskite film fabrication, $\mathrm{PbI}_{2}, \mathrm{PbBr}_{2}, \mathrm{MAI}$, and FAI were dissolved in a mixture of $\gamma$-butyrolactone and $N, N$-dimethylformamide $(4: 6 \mathrm{vol} / \mathrm{vol})$ at $1.2 \mathrm{M}$ and stirred at $60^{\circ} \mathrm{C}$ for $12 \mathrm{~h}$. All these chemicals were purchased from Tokyo Chemical Industry and used without purification. A precursor film was fabricated on a substrate by spin-coating at 4,000 rpm for $30 \mathrm{~s}$. During spin-coating, $0.3 \mathrm{ml}$ of toluene was dropped onto the precursor film. The precursor film was baked on a hot plate at $60^{\circ} \mathrm{C}$ for $15 \mathrm{~min}$, followed by $100^{\circ} \mathrm{C}$ for $30 \mathrm{~min}$, leading to the conversion into the $\mathrm{MA}_{0.6} \mathrm{FA}_{0.4} \mathrm{PbI}_{2 . .8}$ $\mathrm{Br}_{0.2}$ perovskite. The thickness of the perovskite layer was measured at $\approx 300 \mathrm{~nm}$ using a Dektak profilometer (DektakXT, Bruker). The steady-state absorption and PL spectra of perovskite films were measured using absorption (V-730, JASCO) and PL (FP-8300, JASCO) spectrometers. The time-resolved transient $\mathrm{PL}$ decay curves of perovskite films were measured using Hamamatsu Photonics Quantaurus-Tau. Relative PL quantum yields of perovskite films were obtained by measuring the PL intensity at different excitation fluence. Separately, the absolute PL quantum yield of perovskite films was measured at an excitation fluence of $1 \times 10^{-4} \mathrm{~mW} / \mathrm{cm}^{2}$ using an integrating sphere system (Hamamatsu Photonics Quantaurus-QY). Considering this absolute PL quantum yield, the relative PL quantum yields measured at different excitation fluence were converted into the absolute values. The excitation wavelength for all the aforementioned PL measurements was $365 \mathrm{~nm}$.

For the perovskite solar cell fabrication, a thin layer $(\approx 50 \mathrm{~nm})$ of PEDOT:PSS (Clevios, Al4083) was prepared by spin-coating at $3,000 \mathrm{rpm}$ for $45 \mathrm{~s}$ on top of ITO in air, followed by baking the PEDOT:PSS layer at $160^{\circ} \mathrm{C}$ for $10 \mathrm{~min}$. The perovskite layer was prepared in a nitrogen-filled glove box in the same way described earlier. Finally, $30 \mathrm{~nm} \mathrm{C}_{6} 0$, $10 \mathrm{~nm} \mathrm{BCP}$, and $100 \mathrm{~nm}$ Au layers were thermally deposited on top of the perovskite layer under a high vacuum $\left(10^{-4} \mathrm{~Pa}\right)$. The completed solar cells were encapsulated using a glass lid and UV-cured sealant, without exposure to air at all. Current density-voltage and IPCE measurements were performed on the PSCs using a computer-controlled Keithley 2400 source unit and an EQE measurement system (WXS-155S-10: Wacom Denso) under simulated AM1.5G solar illumination from an Xe lamp-based solar simulator (SRO-25 GD, Bunkokeiki). The active area of the solar cells was $16 \mathrm{~mm}^{2}$. The lamp power was carefully calibrated at $100 \mathrm{~mW} \mathrm{~cm}^{-2}$ (1 Sun) using a crystalline Si reference cell with an amorphous Si optical filter (Bunkokeiki), which was certified by the National Institute of Advanced Industrial Science and Technology of Japan. LED properties of solar cells were measured using an integrating sphere system (A10094, Hamamatsu Photonics) equipped with a photonic multichannel analyzer (C9920-12, Hamamatsu Photonics) and a computer-controlled source meter (2400, Keithley). 